\title{
The Role of Fluorine-18-Fluorodeoxyglucose Positron Emission Tomography in Aggressive Histological Subtypes of Thyroid Cancer: An Overview
}

\author{
Giorgio Treglia, ${ }^{1}$ Salvatore Annunziata, ${ }^{2}$ Barbara Muoio, ${ }^{3}$ Massimo Salvatori, ${ }^{2}$ \\ Luca Ceriani, ${ }^{1}$ and Luca Giovanella ${ }^{1}$ \\ ${ }^{1}$ Department of Nuclear Medicine and Thyroid Centre, Oncology Institute of Southern Switzerland, Via Ospedale 12, \\ 6500 Bellinzona, Switzerland \\ ${ }^{2}$ Institute of Nuclear Medicine, Catholic University of the Sacred Heart, Largo Gemelli 8, 00168 Rome, Italy \\ ${ }^{3}$ School of Medicine, Catholic University of the Sacred Heart, Largo Vito 1, 00168 Rome, Italy \\ Correspondence should be addressed to Giorgio Treglia; giorgiomednuc@libero.it
}

Received 9 December 2012; Revised 11 March 2013; Accepted 24 March 2013

Academic Editor: Richard Wahl

Copyright (c) 2013 Giorgio Treglia et al. This is an open access article distributed under the Creative Commons Attribution License, which permits unrestricted use, distribution, and reproduction in any medium, provided the original work is properly cited.

\begin{abstract}
Aggressive histological subtypes of thyroid cancer are rare and have a poor prognosis. The most important aggressive subtypes of thyroid cancer are Hürthle cell carcinoma (HCTC) and anaplastic and poorly differentiated carcinoma (ATC and PDTC). The American Thyroid Association recently published guidelines for the management of patients with ATC, but no specific guidelines have been done about HCTC. We performed an overview of the literature about the role of Fluorine-18-Fluorodeoxyglucose positron emission tomography or positron emission tomography/computed tomography (FDG-PET or PET/CT) in aggressive histological subtypes of thyroid cancer. Only few original studies about the role of FDG-PET or PET/CT in HCTC, PDTC, and ATC have been published in the literature. FDG-PET or PET/CT seems to be useful in staging or followup of invasive and metastatic HCTC. FDG-PET or PET/CT should be used in patients with ATC in initial staging and in the followup after surgery to evaluate metastatic disease. Some authors suggest the use of FDG-PET/CT in staging of PDTC, but more studies are needed to define the diagnostic use of FDG-PET/CT in this setting. Limited experience suggests the usefulness of FDG-PET or PET/CT in patients with more aggressive histological subtypes of DTC. However, DTC presenting as radioiodine refractory and FDG-PET positive should be considered aggressive tumours with poor prognosis.
\end{abstract}

\section{Introduction}

Aggressive histologic subtypes of thyroid cancer are less frequent and have a worse prognosis than well-differentiated thyroid carcinoma (DTC). Most important aggressive subtypes of thyroid cancer are Hürthle cell carcinoma (HCTC) and anaplastic and poorly differentiated carcinoma (ATC and PDTC).

HCTC was firstly considered as subtypes of DTC. Now, it is included in aggressive histologic subtypes, because of its biological behaviour. ATC could be a de novo tumour or arising from dedifferentiation of DTC. During this process, thyroid cancer could be found in an intermediate differentiation pattern, classified as PDTC. Both ATC and PDTC have a poor prognosis and efficient diagnostic tools are needed to improve survival.

After those about DTC and medullary thyroid cancer (MTC) $[1,2]$, American Thyroid Association (ATA) recently published guidelines for the management of patients with ATC [3]. No specific guidelines have been done about HCTC.

Fluorine-18-Fluorodeoxyglucose (FDG), a glucose analogue, is the most used positron emission tomography (PET) tracer in oncology. Although FDG-PET and PET/CT have a moderate sensitivity for early-stage, well-differentiated thyroid malignancy [4], they are currently used in DTC, particularly in postthyroidectomy patients with high serum thyroglobulin $(\mathrm{Tg})$ levels and a negative radioiodine wholebody scan, as prognostic tool in patients with metastases, for 


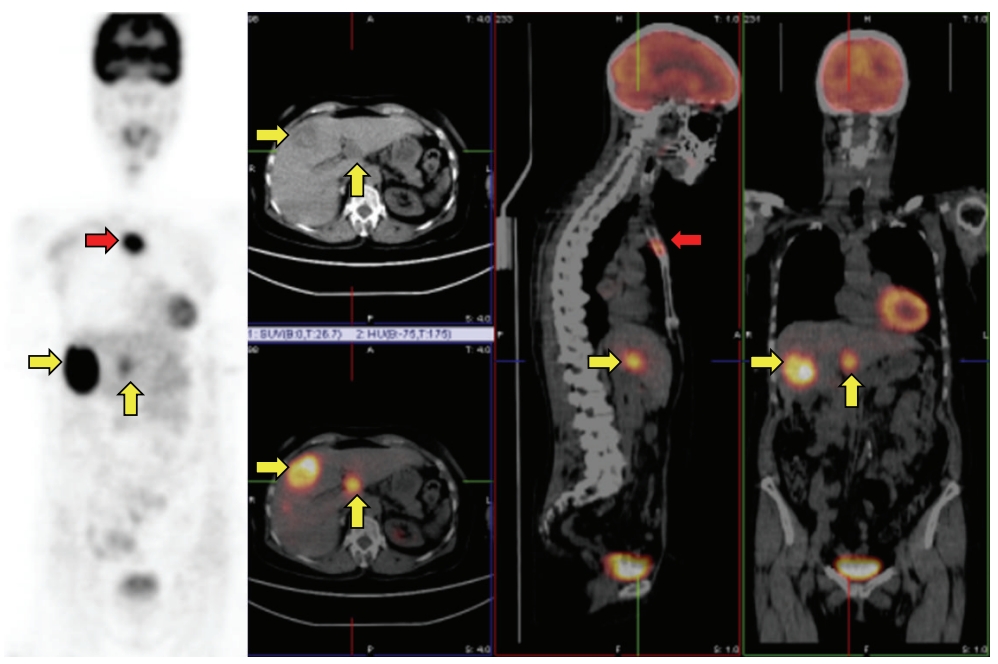

FIGURE 1: FDG-PET/CT in a 68-year-old female previously operated for HCTC showing the presence of sternal (red arrows) and liver metastases (yellow arrows).

the measurement of posttreatment response, and as selection tool in patients not eligible to radioiodine therapy [5-7]. FDG-PET or PET/CT is also used in recurrent MTC and in thyroid nodules with indeterminate or nondiagnostic fine needle aspiration biopsy (FNAB) [5].

During dedifferentiation process (from DTC to ATC), an inverse relationship between radioiodine (I-131) and FDG uptake in thyroid cancer cells was observed (the so-called flip-flop phenomenon) [8]. A recent study highlights that thyroid cancer dedifferentiation is characterized by glucose transporters (GLUT1) upregulation and reduced expression of sodium-iodide symporter (NIS) [9]. This is the rationale to propose FDG-PET or PET/CT as efficient diagnostic tools in ATC, PDTC, and other aggressive subtypes of thyroid cancer.

The aim of this paper is to perform an overview of the literature about the role of FDG-PET or PET/CT in aggressive histological subtypes of thyroid cancer.

\section{FDG-PET in Hürthle Cell Thyroid Carcinoma (HCTC)}

About $3.6 \%$ of thyroid cancers are HCTC [10]. Initially, HCTC was included in DTC group, but it has a different oncogenic expression and is now considered as different histological and clinic disease [11].

HCTC has a 10 -year disease-free survival of $40 \%$ and mortality of $51 \%$ [12], worse than DTC. In fact, HCTC is associated with a high risk of distant and lymph nodal metastases having a worse prognosis compared to DTC.

Only few studies about the role of FDG-PET or PET/CT in HCTC have been published in the literature. Some authors suggested a good sensitivity of FDG-PET in HCTC $[13,14]$ and Hürthle cell adenoma [15]. Overall, HCTC seems to be unable to concentrate I-131, but it is an FDG-avid tumour.

Pryma et al. [16] studied 44 patients with HCTC. There were 24 positive and 20 negative FDG-PET scans giving a sensitivity of $95.8 \%$ and a specificity of $95 \%$. FDG-PET demonstrated a good diagnostic accuracy in HCTC patients. Furthermore, a high FDG uptake was demonstrated to be a negative prognostic factor. These authors suggested that FDG-PET could be indicated in patients with HCTC in postoperative staging and as followup in patients with an increase of $\mathrm{Tg}$ or recurrent disease [16].

Lowe et al. [17] studied 14 FDG-PET scans in patients with HCTC. PET findings were positive in all but 1 of patients with known disease, with a sensitivity of $92 \%$. Moreover, in 7 out of 14 PET scans, a disease not diagnosed by other techniques was demonstrated. In 7 patients, therapy was changed after FDGPET. So, these authors concluded that FDG-PET improves staging and disease management in patients with HTCT [17].

Plotkin et al. [18] evaluated 17 HCTC with FDG-PET. In subgroup A, patients with an elevated Tg level were included $(n=13)$, and in 10 cases PET scans were true positive. In subgroup $B$, patients with a suspect morphologic imaging were included $(n=4)$, and PET scans were true negative in three cases. Only one false positive was found in each group. Overall, FDG-PET demonstrated a sensitivity of $92 \%$, a specificity of $80 \%$, a positive predictive value of $92 \%$, a negative predictive value of $80 \%$, and an accuracy of $89 \%$ in HCTC [18].

Overall, FDG-PET or PET/CT seems to be useful functional imaging methods in initial staging or restaging of HCTC (Figure 1), presenting high diagnostic accuracy in this setting (Table 1).

\section{FDG-PET in Anaplastic Thyroid Carcinoma (ATC)}

ATC is a rare and aggressive tumour, representing less than $5 \%$ of all thyroid carcinomas and originating by thyroid follicular cells (as DTC). ATC is often diagnosed in older patients and usually has a rapid growth and an extensive local invasion [21-23]. Three main histological subtypes of ATC are reported: spindle cell, pleomorphic giant cell, and 
TABLE 1: Main findings about FDG-PET or PET/CT in Hürthle cell thyroid carcinoma, anaplastic thyroid carcinoma, and poorly differentiated thyroid carcinoma.

\begin{tabular}{|c|c|c|c|c|c|c|c|}
\hline Type & Authors & Year & Device & Patients & Sensitivity & Specificity & Comments \\
\hline \multirow{3}{*}{ HCTC } & Pryma et al. [16] & 2006 & $\begin{array}{l}\text { PET or } \\
\text { PET/CT }\end{array}$ & 44 & $95.8 \%$ & $95 \%$ & $\begin{array}{l}\text { FDG-PET has excellent diagnostic accuracy in HCTC, } \\
\text { improving on CT and radioiodine scintigraphy. Intense FDG } \\
\text { uptake is indicator of a poor prognosis. Patients with HCTC } \\
\text { should undergo FDG-PET as part of their initial } \\
\text { postoperative staging and periodically to screen for occult } \\
\text { recurrence, particularly in patients with elevated serum } \\
\text { thyroglobulin }\end{array}$ \\
\hline & Lowe et al. [17] & 2003 & PET & 12 & $91.6 \%$ & N.A. & $\begin{array}{l}\text { HCTC demonstrates intense FDG uptake. PET improves } \\
\text { disease detection and disease management in HCTC relative } \\
\text { to anatomic or radioiodine imaging. FDG-PET should be } \\
\text { recommended for the evaluation and clinical management of } \\
\text { HCTC }\end{array}$ \\
\hline & Plotkin et al. [18] & 2002 & PET & 17 & $100 \%$ & $60 \%$ & $\begin{array}{l}\text { This study supports the efficiency of FDG-PET in the } \\
\text { followup of HCTC }\end{array}$ \\
\hline \multirow{3}{*}{ ATC } & Grabellus et al. [9] & 2012 & $\mathrm{PET} / \mathrm{CT}$ & 4 & $100 \%$ & N.A. & $\begin{array}{l}\text { ATC shows intense FDG uptake. FDG-PET/CT is an } \\
\text { important imaging modality for ATC }\end{array}$ \\
\hline & Poisson et al. [19] & 2010 & $\mathrm{PET} / \mathrm{CT}$ & 20 & $100 \%$ & N.A. & $\begin{array}{l}\text { FDG-PET/CT appears to be the reference imaging modality } \\
\text { for ATC at initial staging and seems promising in the early } \\
\text { evaluation of treatment response and followup }\end{array}$ \\
\hline & Bogsrud et al. [20] & 2008 & PET & 16 & $100 \%$ & N.A. & $\begin{array}{l}\text { FDG-PET may improve disease detection and have an } \\
\text { impact on the management of patients with ATC relative to } \\
\text { other imaging modalities }\end{array}$ \\
\hline PDTC & Grabellus et al. [9] & 2012 & $\mathrm{PET} / \mathrm{CT}$ & 22 & $86.3 \%$ & N.A. & $\begin{array}{l}\text { PDTC shows intermediate FDG uptake between DTC and } \\
\text { ATC. FDG-PET/CT is an important imaging modality for } \\
\text { PDTC }\end{array}$ \\
\hline
\end{tabular}

Legend: N.A.: not available; DTC: differentiated thyroid carcinoma; PDTC: poorly differentiated thyroid carcinoma; ATC: anaplastic thyroid carcinoma; HCTC: Hürthle cell thyroid carcinoma.

squamous cell subtype [3]. In over $70 \%$ of the patients the tumour infiltrates surrounding tissues, and median survival time is about 6-8 months [22]. In differential diagnosis, it is important to distinguish ATC and PDTC. In fact, proportion of ATC, PDTC, or DTC characterizing the thyroid tumour can change prognosis and clinical management [3].

This aggressive thyroid tumour is not able to uptake iodine and to produce $\operatorname{Tg}[19,20,24,25]$. Conversely, ATC has a high glucose metabolism and high FDG uptake [5, 9]. ATA recently published guidelines for management of patients with ATC [3]. ATA recommended FDG-PET and $\mathrm{PET} / \mathrm{CT}$ in evaluating metastatic patients, especially bone lesions. Moreover, FDG-PET may be useful in distinguishing ATC from DTC metastases because of the higher FDG uptake of ATC. Other indications described about FDG-PET or PET/CT in ATC were resectability evaluation and followup, with a higher sensitivity than CT alone. FDG-PET is also recommended 3-6 months after therapy in patients with no disease or in persistent structural disease as a guide to therapy [3].

Few original studies have been published about the role of FDG-PET in ATC (Table 1).

Poisson et al. [19] studied 20 consecutive ATC patients with FDG-PET/CT for initial staging and during followup. Authors analysed progression on imaging followup (CT or PET/CT). Per lesion, organ, and patient analysis have been done. In univariate analysis, maximal standardised uptake value (SUVmax) and functional volume were a predictive factor for survival. Conversely, in bivariate analysis, only functional volume was a prognostic factor. Early evaluation of treatment has been done in 4 out of 11 patients in whom PET and CT were both registered. After treatment with combined radiotherapy and chemotherapy, a negative FDG$\mathrm{PET} / \mathrm{CT}$ scan confirmed a complete long-term remission. Finally, authors suggested the use of FDG-PET/CT in ATC during initial staging. Among other imaging modalities, only preoperative CT should be requested. FDG-PET/CT could be also recommended in both early and long-term followup and in the assessment of treatment response [19].

Bogsrud et al. [20] investigated the role of FDG-PET in the management of patients with ATC. PET data were compared with other diagnostic tools (CT, ultrasound, magnetic resonance imaging, bone scan, and histology) and with clinical follow-up. In all 16 patients included, PET records resulted true positive for primary tumours. In $50 \%$ of patients, PET data influenced the clinical management. These authors concluded that FDG-PET could improve disease staging changing the clinical management of patients with ATC [20].

Overall, FDG-PET or PET/CT should be used in patients with ATC in initial staging and in the followup after surgery to evaluate metastatic disease (Figure 2). In selected cases, these functional imaging methods may be helpful in directing treatment and in evaluating the efficacy of therapy. New 

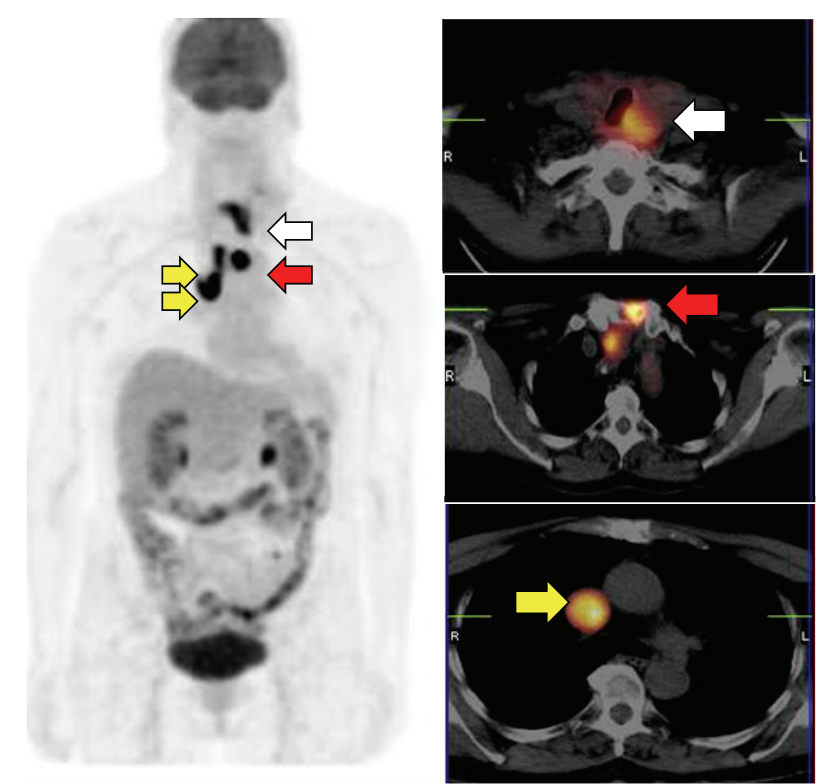

FIGURE 2: FDG-PET/CT in a 58-year-old female with ATC showing the presence of increased uptake in the thyroid tumour (white arrow) and sternal (red arrows) and mediastinal lymph nodal metastases (yellow arrows).

studies are needed to investigate the role of FDG-PET or PET/CT in detecting the proportion of DTC, ATC, and PDTC in the same tumour.

\section{FDG-PET in Poorly Differentiated Thyroid Carcinoma (PDTC)}

PDTC is an intermediate histological subtype between DTC and ATC and may be a transition form. Conversely to ATC, PDTC preserves some markers of differentiation, such as Tg and thyroid transcription factor 1 (TTF1), and does not represent a de novo tumour [26]. In dedifferentiation process, PDTC and ATC present a growing number of chromosomal alteration, such as RAS, BRAF, TP53, and b-catenin mutations [3, 11]. Activation of the PTEN/PI3 kinase/Akt/mammalian target of rapamycin pattern and mutation of the AKT or PIK3CA genes are more common in PDTC and ATC than DTC [26-29]. These metabolic pathways could be related to the different FDG-PET pattern in different subtypes of thyroid cancer.

PDTC has an intermediate GLUT1 expression and FDG uptake between ATC and DTC, because of "flip-flop" phenomenon [9, 30]. More often PDTC is an FDG-PET positive tumour $[5,9]$. An in vitro study suggested that thyrotropin (TSH) increases FDG uptake in PDTC cells; so, FDG-PET scans under TSH stimulation may be more efficient [31]. Surgery and radiotherapy could be indicated in treatment of PDTC but not radioiodine treatment, because of poor radioiodine uptake.

No studies analysed the role of FDG-PET in PDCT only. Some authors suggested the use of FDG-PET or PET/CT in

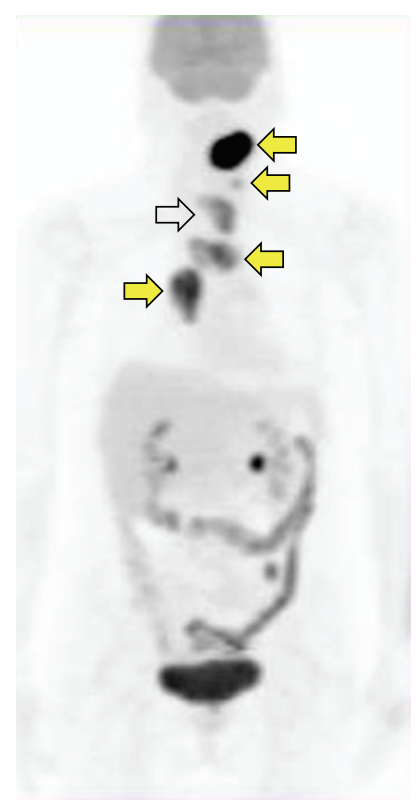

FIGURE 3: FDG-PET in a 48-year-old female with PDTC showing the presence of increased uptake in the thyroid tumour (white arrow) and multiple cervical and mediastinal lymph nodal metastases (yellow arrows).

staging patients with PDTC (Figure 3), especially in postthyroidectomy staging of high-risk patients [11]. Some authors studied the role of FDG-PET in all thyroid cancer subtypes but included only few cases of PDTC in their analysis, not sufficient to conclude that FDG-PET is efficient for this histological tumour type.

More preclinical and clinical studies are needed about FDG-PET or PET/CT in PDTC to demonstrate the clinical usefulness of FDG-PET in PDTC.

\section{FDG-PET in More Aggressive Histological Subtypes of DTC}

Limited experience exists about the role of FDG-PET or $\mathrm{PET} / \mathrm{CT}$ in patients with more aggressive histological subtypes of DTC, including case reports or small case series in patients with tall cell [32], diffuse sclerosing [33-35], solid/trabecular [36] and insular variant [37] of DTC. These articles underlines that FDG-PET or PET/CT seem to be very useful tools for the staging and restaging of such tumours.

\section{Noniodine Concentrating Metastases of DTC}

Radioactive iodine-refractory (RAIR) FDG-PET positive thyroid carcinomas represent the major cause of deaths from thyroid carcinomas and are therefore the main focus of novel target therapies. Although the majority of primary thyroid carcinomas leading to RAIR FDG-PET positive metastatic disease are PDTC, DTC can also be responsible for RAIR disease. Histologic characterization of metastases/recurrence 
in 70 RAIR FDG-PET positive thyroid carcinoma patients revealed that $47.1 \%$ had PDTC, $20 \%$ had tall-cell variant of papillary thyroid carcinoma, $22.9 \%$ had well-differentiated papillary thyroid carcinoma (including classic and follicular variants), $8.6 \%$ had HCTC, and $1.4 \%$ had ATC [30].

DTC presenting FDG uptake on PET scan and histological features such as necrosis should be considered aggressive differentiated cancers and FDG uptake in these tumours is highly prognostic for survival [38].

\section{Conclusions}

From this overview of the literature about the usefulness of FDG-PET or PET/CT in aggressive subtypes of thyroid tumours, we conclude the following:

(i) the role of FDG-PET or PET/CT in patients with HCTC is clear in initial staging or followup of invasive and metastatic tumours;

(ii) FDG-PET or PET/CT is recommended in staging, followup, and posttreatment restaging of ATC, especially in metastatic disease, as published in ATA guidelines;

(iii) further evaluations are needed to investigate the role of FDG-PET or PET/CT in PDTC, because of the difficulties connected to define the biological behaviour of this aggressive subtype of thyroid cancer;

(iv) limited experience suggests the usefulness of FDGPET or PET/CT in patients with more aggressive histological subtypes of DTC;

(v) DTC presenting as RAIR and FDG-PET positive should be considered aggressive tumours with poor prognosis.

\section{Conflict of Interests}

The Authors declare no conflict of interests.

\section{References}

[1] D. S. Cooper, G. M. Doherty, B. R. Haugen et al., "Revised American thyroid association management guidelines for patients with thyroid nodules and differentiated thyroid cancer," Thyroid, vol. 19, no. 11, pp. 1167-1214, 2009.

[2] R. T. Kloos, C. Eng, D. B. Evans et al., "Medullary thyroid cancer: management guidelines of the American thyroid association," Thyroid, vol. 19, no. 6, pp. 565-612, 2009.

[3] R. C. Smallridge, K. B. Ain, S. L. Asa et al., "American association guidelines for management of patients with anaplastic thyroid cancer," Thyroid, vol. 22, no. 11, pp. 1104-1139, 2012.

[4] F. Grünwald, T. Kälicke, U. Feine et al., "Fluorine-18 fluorodeoxyglucose positron emission tomography in thyroid cancer: results of a multicentre study," European Journal of Nuclear Medicine, vol. 26, no. 12, pp. 1547-1552, 1999.

[5] G. Treglia, B. Muoio, L. Giovanella, and M. Salvatori, “The role of positron emission tomography and positron emission tomography/computed tomography in thyroid tumours: an overview," European Archives of Oto-Rhino-Laryngology, 2012.
[6] L. Giovanella, "Positron emission tomography/computed tomography in patients treated for differentiated thyroid carcinomas," Expert Review of Endocrinology and Metabolism, vol. 7, no. 1, pp. 35-43, 2012.

[7] L. Giovanella, L. Ceriani, D. de Palma, S. Suriano, M. Castellani, and F. A. Verburg, "Relationship between serum thyroglobulin and 18FDG-PET/CT in 131I-negative differentiated thyroid carcinomas," Head and Neck, vol. 34, no. 5, pp. 626-631, 2012.

[8] U. Feine, R. Lietzenmayer, J. P. Hanke, H. Wohrle, and W. Muller-Schauenburg, "18FDG whole-body PET in differentiated thyroid carcinoma. Flipflop in uptake patterns of 18 FDG and 131I," NuklearMedizin, vol. 34, no. 4, pp. 127-134, 1995.

[9] F. Grabellus, J. Nagarajah, A. Bockisch, K. W. Schmid, and S. Y. Sheu, "Glucose transporter 1 expression, tumor proliferation, and iodine/glucose uptake in thyroid cancer with emphasis on poorly differentiated thyroid carcinoma," Clinical Nuclear Medicine, vol. 37, no. 2, pp. 121-127, 2012.

[10] S. A. Hundahl, B. Cady, M. P. Cunningham et al., "Initial results from a prospective cohort study of 5583 cases of thyroid carcinoma treated in the United States during 1996. U.S. and German thyroid cancer study group: an American college of surgeons commission on cancer patient care evaluation study," Cancer, vol. 89, no. 1, pp. 202-217, 2000.

[11] T. Abraham and H. Schöder, "Thyroid cancer-indications and opportunities for positron emission tomography/computed tomography imaging," Seminars in Nuclear Medicine, vol. 41, no. 2, pp. 121-138, 2011.

[12] A. Stojadinovic, R. A. Ghossein, A. Hoos et al., "Hürthle cell carcinoma: a critical histopathologic appraisal," Journal of Clinical Oncology, vol. 19, no. 10, pp. 2616-2625, 2001.

[13] C. L. Blount and H. J. Dworkin, "F-18 FDG uptake by recurrent Hurthle cell carcinoma of the thyroid using high-energy planar scintigraphy," Clinical Nuclear Medicine, vol. 21, no. 11, pp. 831833, 1996.

[14] W. Wiesner, H. Engel, G. K. von Schulthess, G. P. Krestin, and I. Bicik, "FDG PET-negative liver metastases of a malignant melanoma and FDG PET-positive Hurthle cell tumor of the thyroid," European Radiology, vol. 9, no. 5, pp. 975-978, 1999.

[15] B. H. Lang, "The role of 18 F-fluorodeoxyglucose positron emission tomography in the prognostication, diagnosis, and management of thyroid carcinoma," Journal of Thyroid Research, vol. 2012, Article ID 198313, 8 pages, 2012.

[16] D. A. Pryma, H. Schöder, M. Gönen, R. J. Robbins, S. M. Larson, and H. W. D. Yeung, "Diagnostic accuracy and prognostic value of 18F-FDG PET in Hürthle cell thyroid cancer patients," Journal of Nuclear Medicine, vol. 47, no. 8, pp. 1260-1266, 2006.

[17] V. J. Lowe, B. P. Mullan, I. D. Hay, B. McIver, and J. L. Kasperbauer, "18F-FDG PET of patients with Hürthle cell carcinoma," Journal of Nuclear Medicine, vol. 44, no. 9, pp. 1402-1406, 2003.

[18] M. Plotkin, H. Hautzel, B. J. Krause et al., "Implication of 218fluor-2-deoxyglucose positron emission tomography in the follow-up of Hürthle cell thyroid cancer," Thyroid, vol. 12, no. 2, pp. 155-161, 2002.

[19] T. Poisson, D. Deandreis, S. Leboulleux et al., "18F-fluorodeoxyglucose positron emission tomography and computed tomography in anaplastic thyroid cancer," European Journal of Nuclear Medicine and Molecular Imaging, vol. 37, no. 12, pp. 2277-2285, 2010.

[20] T. V. Bogsrud, D. Karantanis, M. A. Nathan et al., "18F-FDG PET in the management of patients with anaplastic thyroid carcinoma," Thyroid, vol. 18, no. 7, pp. 713-719, 2008. 
[21] C. Are and A. R. Shaha, "Anaplastic thyroid carcinoma: biology, pathogenesis, prognostic factors, and treatment approaches," Annals of Surgical Oncology, vol. 13, no. 4, pp. 453-464, 2006.

[22] S. Chiacchio, A. Lorenzoni, G. Boni, D. Rubello, R. Elisei, and G. Mariani, "Anaplastic thyroid cancer: prevalence, diagnosis and treatment," Minerva Endocrinologica, vol. 33, no. 4, pp. 341-357, 2008.

[23] B. McIver, I. D. Hay, D. F. Giuffrida et al., "Anaplastic thyroid carcinoma: a 50-year experience at a single institution," Surgery, vol. 130, no. 6, pp. 1028-1034, 2001.

[24] N. Khan, N. Oriuchi, T. Higuchi, and K. Endo, "Review of fluorine-18-2-fluoro-2-deoxy-d-glucose positron emission tomography (FDG-PET) in the follow-up of medullary and anaplastic thyroid carcinomas," Cancer Control, vol. 12, no. 4, pp. 254-260, 2005.

[25] C. Mosci and A. Iagaru, "PET/CT imaging of thyroid cancer," Clinical Nuclear Medicine, vol. 36, no. 12, pp. e180-e185, 2011.

[26] Y. E. Nikiforov, "Genetic alterations involved in the transition from well-differentiated to poorly differentiated and anaplastic thyroid carcinomas," Endocrine Pathology, vol. 15, no. 4, pp. 319327, 2004.

[27] G. Garcia-Rostan, H. Zhao, R. L. Camp et al., "ras Mutations are associated with aggressive tumor phenotypes and poor prognosis in thyroid cancer," Journal of Clinical Oncology, vol. 21, no. 17, pp. 3226-3235, 2003.

[28] M. Volante, I. Rapa, M. Gandhi et al., "RAS mutations are the predominant molecular alteration in poorly differentiated thyroid carcinomas and bear prognostic impact," Journal of Clinical Endocrinology and Metabolism, vol. 94, no. 12, pp. 47354741, 2009.

[29] W. W. Ma, H. Jacene, D. Song et al., "[18F]fluorodeoxyglucose positron emission tomography correlates with Akt pathway activity but is not predictive of clinical outcome during mTOR inhibitor therapy," Journal of Clinical Oncology, vol. 27, no. 16, pp. 2697-2704, 2009.

[30] M. Rivera, R. A. Ghossein, H. Schoder, D. Gomez, S. M. Larson, and R. M. Tuttle, "Histopathologic characterization of radioactive iodine-refractory fluorodeoxyglucose-positron emission tomography-positive thyroid carcinoma," Cancer, vol. 113, no. 1, pp. 48-56, 2008.

[31] C. H. Kim, I. R. Yoo, Y. A. Chung et al., "Influence of thyroidStimulating hormone on $18 \mathrm{~F}$-fluorodeoxyglucose and $99 \mathrm{mTc}-$ methoxyisobutylisonitrile uptake in human poorly differentiated thyroid cancer cells in vitro," Annals of Nuclear Medicine, vol. 23, no. 2, pp. 131-136, 2009.

[32] R. Ghossein and V. A. Livolsi, "Papillary thyroid carcinoma tall cell variant," Thyroid, vol. 18, no. 11, pp. 1179-1181, 2008.

[33] C. S. Kuo, K. T. Tang, J. D. Lin, A. H. Yang, C. H. Lee, and H. D. Lin, "Diffuse sclerosing variant of papillary thyroid carcinoma with multiple metastases and elevated serum carcinoembryonic antigen level," Thyroid, vol. 22, no. 11, pp. 1187-1190, 2012.

[34] Y. H. Xu, H. J. Song, Z. L. Qiu, and Q. Y. Luo, "Extensive lymph node metastases found by (18)F-FDG-PET/CT in a patient with diffuse sclerosing variant of papillary thyroid carcinoma," Hellenic Journal of Nuclear Medicine, vol. 14, no. 2, pp. 188-189, 2011.

[35] T. Z. Wong, M. K. Jain, and S. E. Spratt, "I-131, I-123, and F-18 FDG-PET imaging in a patient with diffuse sclerosing variant of papillary thyroid cancer," Clinical Nuclear Medicine, vol. 33, no. 12 , pp. 834-837, 2008.

[36] L. Giovanella, F. Fasolini, S. Suriano, and L. Mazzucchelli, "Hyperfunctioning solid/trabecular follicular carcinoma of the thyroid gland," Journal of Oncology, vol. 2010, Article ID 635984, 4 pages, 2010.

[37] M. Diehl, S. Graichen, C. Menzel, E. Lindhorst, and F. Grünwald, "F-18 FDG PET in insular thyroid cancer," Clinical Nuclear Medicine, vol. 28, no. 9, pp. 728-731, 2003.

[38] D. Deandreis, A. Al Ghuzlan, S. Leboulleux et al., "Do histological, immunohistochemical, and metabolic (radioiodine and fluorodeoxyglucose uptakes) patterns of metastatic thyroid cancer correlate with patient outcome?" Endocrine-Related Cancer, vol. 18, no. 1, pp. 159-169, 2011. 


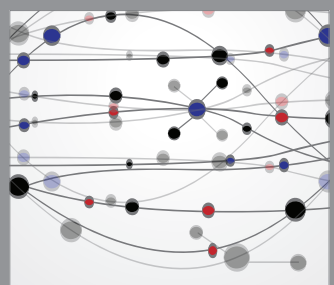

The Scientific World Journal
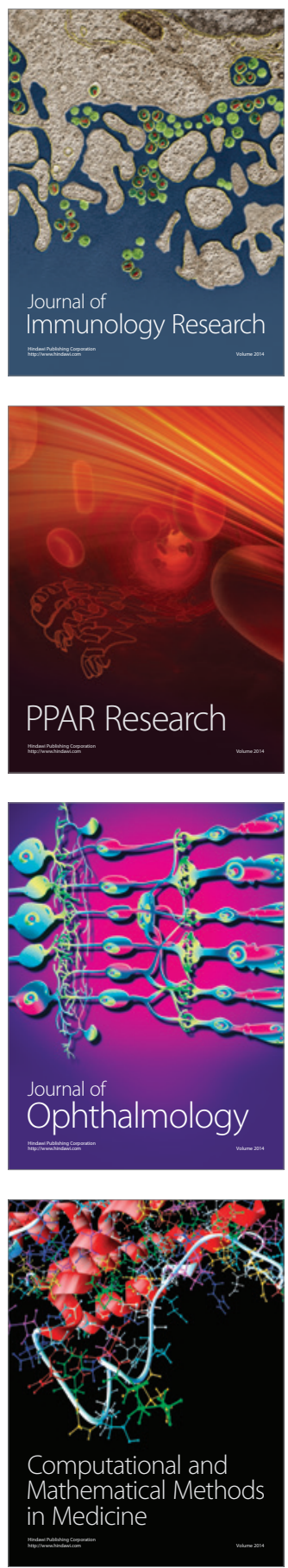

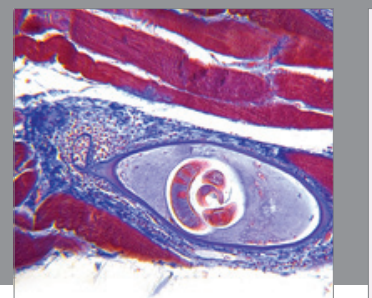

Gastroenterology

Research and Practice
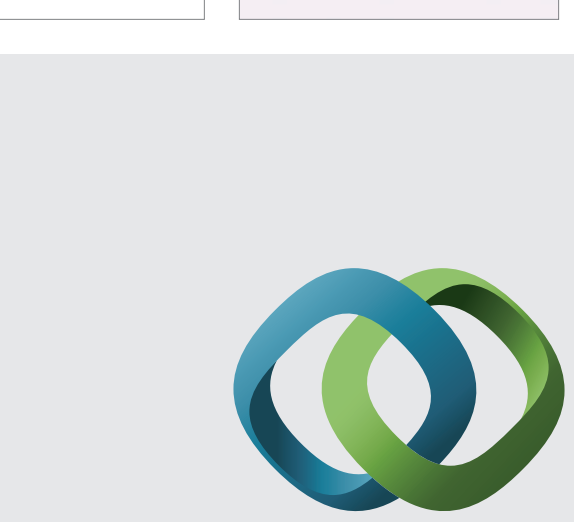

\section{Hindawi}

Submit your manuscripts at

http://www.hindawi.com
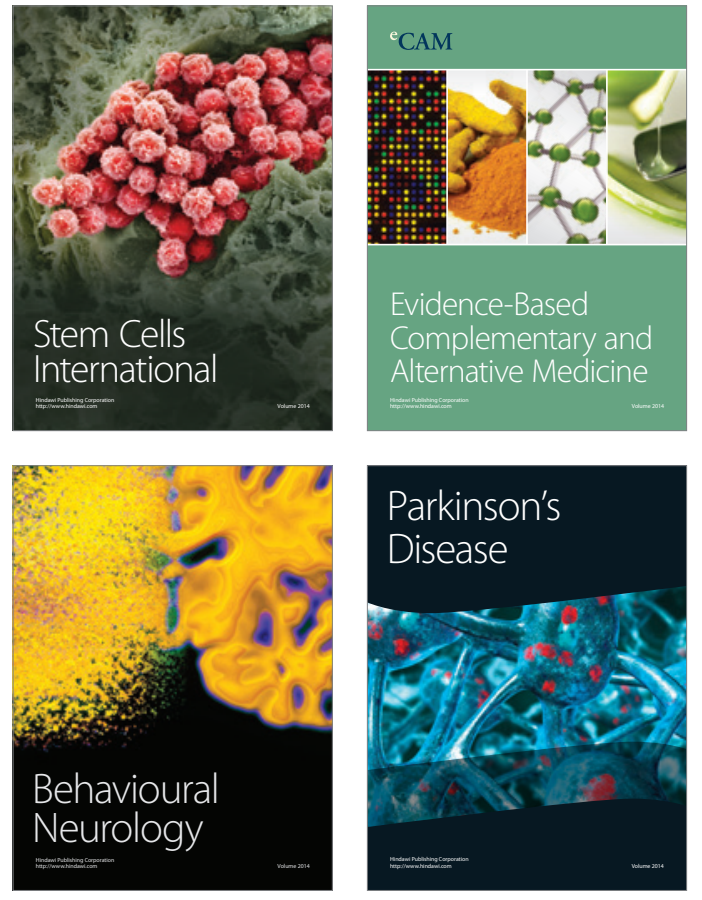
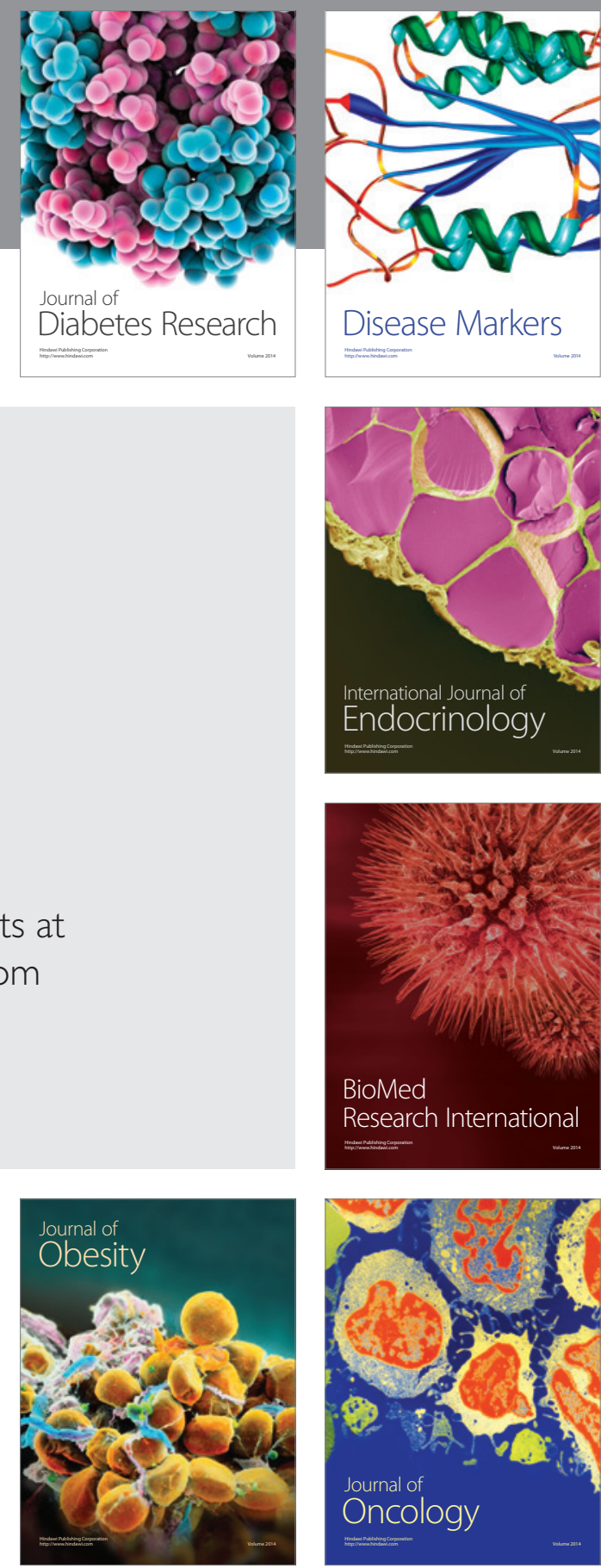

Disease Markers
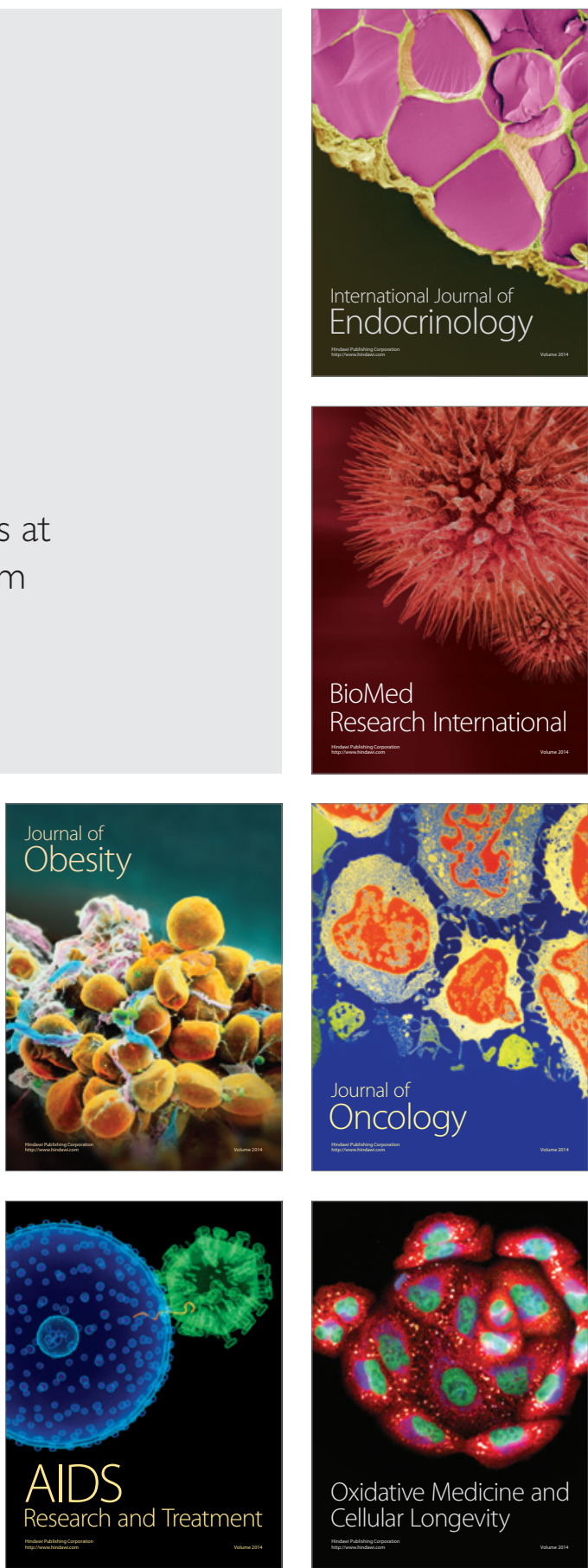